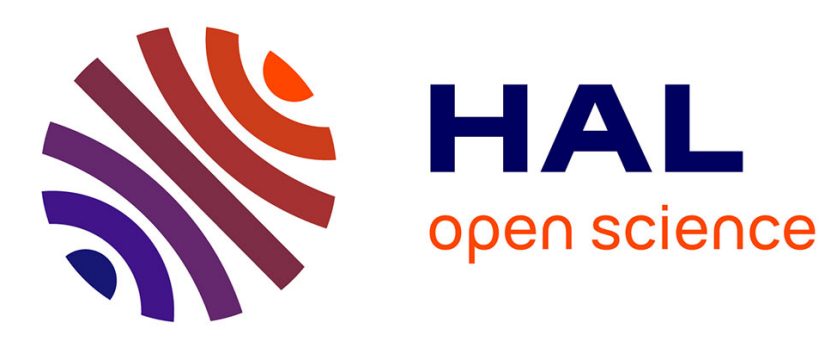

\title{
A DEVS/MAS-based Framework for Modeling/Simulation of Complex Systems
}

Noureddine / Seddari, Mohammed Redjimi, Mohamed Belaoued

\section{To cite this version:}

Noureddine / Seddari, Mohammed Redjimi, Mohamed Belaoued. A DEVS/MAS-based Framework for Modeling/Simulation of Complex Systems. Advanced Intelligent Systems for Sustainable Development (AI2SD'2019), Jul 2019, Marrakech, Morocco. hal-02421679

\section{HAL Id: hal-02421679 https://hal.science/hal-02421679}

Submitted on 20 Dec 2019

HAL is a multi-disciplinary open access archive for the deposit and dissemination of scientific research documents, whether they are published or not. The documents may come from teaching and research institutions in France or abroad, or from public or private research centers.
L'archive ouverte pluridisciplinaire HAL, est destinée au dépôt et à la diffusion de documents scientifiques de niveau recherche, publiés ou non, émanant des établissements d'enseignement et de recherche français ou étrangers, des laboratoires publics ou privés. 


\title{
A DEVS/MAS-based Framework for Modeling/Simulation of Complex Systems
}

\author{
Noureddine Seddari ${ }^{1,2}$, Mohammed Redjimi ${ }^{1}$, and Mohamed Belaoued ${ }^{2}$ \\ ${ }^{I}$ Department of Computer Science, 20 August 1955 University of Skikda, Algeria. \\ ${ }^{2}$ LIRE Laboratory, Software Technologies and Information Systems Department, \\ University of Abdelhamid Mehri, Constantine, Algeria. \\ n. seddari@univ-skikda.dz \\ ${ }^{1}$ medredjimilgmail.com \\ ${ }^{2}$ belaoued.mohamedegmail.com
}

\begin{abstract}
This paper presents an approach for modeling and simulation of complex systems. This approach is based on the decomposition of the considered systems into sub-systems which appear on two levels: On the lower level; the decomposition concerns the division of a global system into atomic and coupled models based on DEVS formalism (Discrete Event Systems Specification). The system components are then represented using the DEVS mathematical equations. This step allows the formal system checking. At the higher lever, the implementation of the obtained DEVS models is realized using Multi-Agents Systems (MAS) based on Agent/Role/Group (AGR). Moreover, a set of procedures and functions permitting to transform DEVS to MAS models are defined and implemented. The main advantages of this approach are its adaptability for various domains, its flexibility (easy to implement), its extensibility (adding new components). A version of this work will be implemented using a functional extension of the Multi Agent Development KIT platform (MAD-KIT).
\end{abstract}

Keywords: Complex Systems, Discrete Event Systems Specification (DEVS), Modeling and Simulation, Multi-Agent Systems (MAS), AGR Model (Agent/Group/Role), MAD-KIT.

\section{Introduction}

Nowadays, complex systems are made of a large number of interacting entities into interaction, and the overall behavior of these systems emerges from these interactions $[1,2]$. The increasing complexity of the studied systems and the requirements of the designers have led to the introduction of many categories of formalisms into the modelling and simulation process. Indeed, there is no single model, or formalism, to model all aspects or components of a system. Thus, different levels of explanation, different theories, and different formalisms or dedicated languages are necessary to properly describe the different aspects or components of the system.

The modeling and simulation [3-10] of complex systems can be used for several purposes. For instance, in ca be used in order to handle the modeled system without risks, to observe and analyze the phenomena emerging when we change the system inputs, and also to improve the understanding and to prevent eventual damages of the system. Therefore, the challenge of the modeling and simulation process of complex 
systems is to provide adequate formalism to model these systems on one hand, and to use the appropriate tools for implementation, on the other hand.

This work presents a hybrid method that combines DEVS (Discrete Event System Specification) formalism [11] and AGR model (Agent/Group/Role) for modeling and simulating complex systems.

First, DEVS formalism [11] is used to define the structures of entities that make up the system by using both atomic and coupled models. The DEVS representation encapsulates the structure and the behavior of the model. Thus, DEVS models provide mathematical representations that can be formally checked and validated.

Second, multi-agent systems (MAS) present a strong theoretical and practical tool to implement the solution and provide several advantages such as autonomy, proactivity, distribution, self-organization and adaptation. The agents cooperate for collective problem solving, since an isolated agent cannot solve the entire problem because it has not large perception and capabilities. Therefore, the system of agents provides a collective solution that emerges from collective actions within the population of the agents.

The idea developed here is focused on the transformation of models checked and validated in DEVS to agent model representation. The Agent/Role/Group (AGR) concept presents the most convenient paradigm in this scope. Moreover, a set of procedures allowing transition between DEVS model and AGR model has been determined and integrated in MAD-KIT platform. The latter has been developed by the team of Professor J. Ferber in LIRMM [12] and based on the principles of AGR.

This paper is organized as follows: The second and third sections present the MultiAgent Systems and DEVS formalism. The fourth section describes related works, where we categorize combinations of DEVS and MAS from which our approach was inspired. Both approach and algorithms transformations that we propose are shown in the fifth section. Finally, section 6 concludes the work and underlines our perspectives.

\section{The Multi-Agent Systems (MAS)}

J. Ferber [13] [14] [15] represents a multi-agent system by the couple $\langle A, W\rangle$ where $\mathrm{A}$ is an agent and $\mathrm{W}$ an environment, and considers an agent in interaction with the world as a system composed of dynamic coupled two subsystems, the coupling taking place through perceptions that the agent has for environment and actions that modify this environment.

$$
\begin{gathered}
A=\left(P_{a}, \operatorname{Percept}_{a}, F_{a}, \operatorname{Infl}_{a}, S_{a}\right) \\
W=(E, \Gamma, \Sigma, R)
\end{gathered}
$$

With

- $\mathrm{P}_{\mathrm{a}}$ represents the function of perception of the agent,

- Percept ${ }_{\mathrm{a}}$ the set of stimuli and sensations,

- $F_{a}$ the function of behavior of the agent that determines the agent's state,

- Infl $\mathrm{a}_{\mathrm{a}}$ the function of action of the agent,.

$-S_{a}$ the set of the agent's internal states, 
- E the space in which the agent evolves,

- $\Gamma$ the space of influences produced by the agent and having like consequences to modify the evolution of the environment,

$-\Sigma$ the set of states of the environment,

- $\mathrm{R}$ the law of evolution of the environment,

\subsection{AGR model}

The model (AGR) is based on the concepts of agents, groups and roles [12] [16].

Agent. An agent is only specified as an active communicating entity which plays roles within groups.

Group. Groups are defined as atomic sets of agent aggregation representing any usual multi-agent system. Each agent is part of one or more group.

Role. The role is abstract representation of an agent function, service or identification within a group. Each role handled by an agent is local to a group [17-20]. Figure 1 represents the diagram of AGR model.

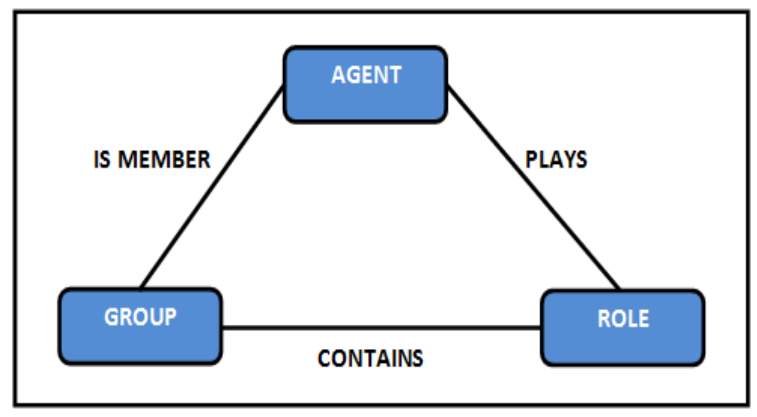

Fig. 1. AGR model

\section{The DEVS Formalism}

The Discrete Event System Specification (DEVS) [3] [21] is an environment for systems' modeling and simulation. DEVS provides a means of specifying a mathematical object called a system. In addition, DEVS allows gathering, in coherent way, other formalisms of modeling. It is, in fact, a formalism fitted to significant number of scopes of application [22-25].

\subsection{Formal Specification of a DEVS Atomic Model}

A Discrete Event System Specification is a structure

$$
\operatorname{ADEVS}=\left(\mathrm{X}, \mathrm{Y}, \mathrm{S}, \delta_{\text {int }}, \delta_{\mathrm{ext}}, \delta_{\mathrm{con}}, \mathrm{t}_{\mathrm{a}}, \lambda\right)
$$

Where

$\mathrm{X}$ is the set of inputs

$\mathrm{S}$ is a set of states

$\mathrm{Y}$ is the set of outputs

$\delta_{\text {int }}: \mathrm{S} \rightarrow \mathrm{S}:$ is the internal transition function 
$\delta_{e x t}: \mathrm{Q} \times \mathrm{X} \rightarrow \mathrm{S}:$ is the external transition function, where

$\mathrm{Q}=\{(\mathrm{s}, \mathrm{e}) \mid \mathrm{s} \in \mathrm{S}, 0 \leq \mathrm{e} \leq \mathrm{ta}(\mathrm{s})\}$ is the total state set

$\mathrm{e}$ is the time elapsed since last transition.

$\lambda: \mathrm{S} \rightarrow \mathrm{Y}$ is the output function

$\mathrm{t}_{\mathrm{a}}(\mathrm{s})$ is the time advance function

\subsection{Formal Specification of a DEVS Coupled Model}

A DEVS coupled model is described by the following equation:

$$
\text { CDEVS }=\left(\mathrm{X}_{\text {self }}, \mathrm{Y}_{\text {self }}, \mathrm{D},\{\mathrm{Md} / \mathrm{d} \in \mathrm{D}\}, \mathrm{EIC}, \mathrm{EOC}, \mathrm{IC}\right)
$$

Self: is the model itself.

$\mathrm{X}_{\text {self }}$ is the set of inputs of coupled model.

$\mathrm{Y}_{\text {self }}$ is the set of outputs of coupled model.

$\mathrm{D}$ is the set of names associated with the components of the model, self is not in D.

$\{\mathrm{Md} / \mathrm{d} \in \mathrm{D}\}$ is the set of components of coupled model.

EIC, EOC and IC define the coupling structure in coupled model.

EIC is the set of external input couplings.

EOC is the external output couplings.

IC defines internal coupling.

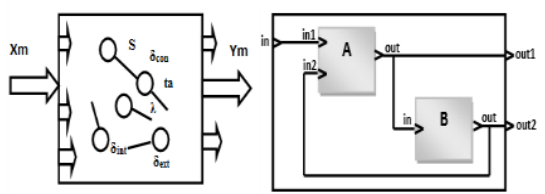

Fig. 2. Graphical representation of an atomic model and a coupled model consisting of two atomic models (A) and (B)

\section{$4 \quad$ Related works}

In this section, we presnt the most relevet tools and platforms for systems modeling and simulation frameworks that combined both DEVS and MAS.

The GALATEA platform [26] [27] provides a high level simulation concepts for specification and design of MAS systems, Thus, one can modeling roles and action plans and establishing communications, negotiations and dialogues between agents to allowing the emergence of efficiency distributed solutions. GALATEA is based on the Zeigler theory of simulation and the logical agents. This platform uses a set of logical programming oriented languages and allows the modeling by using DEVS formalisms. The major disadvantage of this platform is its difficulty of implementation [28].

The JAMES platform [29-31] allows dynamic simulations of MAS systems. An agent is represented by atomic DEVS model, a group of agents is represented by coupled DEVS model and the communication between models is determined by the 
inputs and outputs events. The agent environment plays an important role and it is perceived as a shared framework for the agents interactions [32-35].

The VLE platform [36-37] is a high level simulation framework based on DEVS formalism. This platform allows the simulation of the MAS systems by using DEVS. An agent is represented by a DEVS atomic model, an external stimulus by a message and the environment is characterized by CELL-DEVS [38-39].

The DEVSimPy platform [34] considers the advantages and the disadvantages of these latter platforms and provides a framework for agents modeling and simulation by using DEVS formalism. Thus, both the agents and the environments are represented by DEVS atomic models. A group of agents is represented by a DEVS coupled model [40].

By analyzing the aforementioned works, one can conclude that in these approaches; the agents, the environments, the interactions between agents and the groups of agents are represented by atomic and coupled DEVS models.

Multi-agent systems (MAS), for their part, present a strong theoretical and practical tool in the field of simulation. MAS include tools for managing the agents. At this level, the implementation of the system can be easily realized. For these reasons, we opted in our work for the combination

\section{$5 \quad$ Proposed approach}

In this work, we propose a new approach for modeling and simulation of complex systems and precisely industrial ones. After studying the most relevant existing approaches we distinguished the following correspondence between DEVS and AGR models :

- A DEVS atomic model can be represented by agent model. The inputs and outputs of the atomic models are represented by a set of stimuli and action of the agent. The internal transition function and the external transition function are represented by the function of behaviour and the function of perception of the agent respectively. The output function represented by the function of action of the agent. The sequences of states (S) of the DEVS atomic model are by the set of the agent's internal states.

- A coupled model materializes a group. The inputs and outputs of coupled model are represented by transmitter coordinator agents. The external input couplings, the external output couplings and the internal coupling are represented by different interconnections between agents and groups of agents.

According to the aforementioned points, we provide three major contributions:

- Industrial systems are classified as discrete event dynamic systems (DEDS) and are characterized by a complex and hierarchical structure [41]. In our approach, the structure of industrial system's elements is formally defined and represented in DEVS, and according to B.P. Zeigler [11] we can prove that this formalism is adequate to model of this type of systems.

- Providing a mechanism which can systematically transform the DEVS to AGR model.

- Integrating this mechanism in MAD-KIT platform in order to create a new extension for DEVS formalism. Choosing MAD-KIT platform for implementation can be justified by its advantages such as, its genericity, its independence to a specific field of application, and finally, its simplicity of implementation. 


\subsection{Mechanisms of DEVS to AGR transformation}

The proposed Algorithm below transforms atomic and coupled model of DEVS model to agent and groups of AGR model [41].

The CDEVS_G (CDEVS) function creates the groups Gi, then, the procedures CDEVS_EIC,CDEVS_IC and CDEVS_EOC define the various interconnections between the agents (AGAj "agent corresponding to atomic model ADEVSj" created by ADEVS AGA (ADEVSj) function, AGCi R "Agent representing the input ports of coupled model CDEVSi" and AGCi_E "Agent representing the output ports of coupled model CDEVSi ") and the groups Gi according to coupling type CDEVSi as shown below.

The ADEVS_AGA (ADEVS) function allows the creation of AGAj agents for each atomic model ADEVSj. Thus, both receptors and effectors of AGAj agent represent respectively the input ports (ADEVSj. Inportsi) and output ports (ADEVSj.Outportsi) of atomic model. The function of perception AGAj.Pa corresponds to the external transition function ADEVSj. .ext.

The behavior function AGAj.Fa represents the internal transition function ADEVSj.dint. The action function of AGAj.Infla agent corresponds to the output function ADEVSj. $\lambda$ The dynamic evolution of an agent represents time advance function ADEVSj.ta and the internal states of AGAj agent. This corresponds to the set of states ADEVSj.S.

The CDEVS_AGC (CDEVSj) function creates two coordinators agents AGC for each coupled model CDEVSi, the first one is a receiver coordinator agent (AGCi_R) and the second one is a transmitter coordinator agent (AGCi_E). The main objective of those both coordinators is well to conserve the encapsulation of DEVS formalism. The model is represented as a black box that acts with environment only via the input and output ports .However, the agents have free interaction with their environment. In this approach; the agents want to communicate with internal agents of a group corresponding to a DEVS coupled model should necessary go through coordinators agents of this group.

The CDEVS_G ( CDEVSi ) function creates groups Gi for each CDEVSi coupled model; then it creates AGAj agents that it will gather them in this group Gi. Then; it creates both coordination agents AGCi_R and AGCi_E through CDEVS_AGC function. The figure 3 below illustrate a graphical representation of elementary transformation of our approach DEVS/AGR.

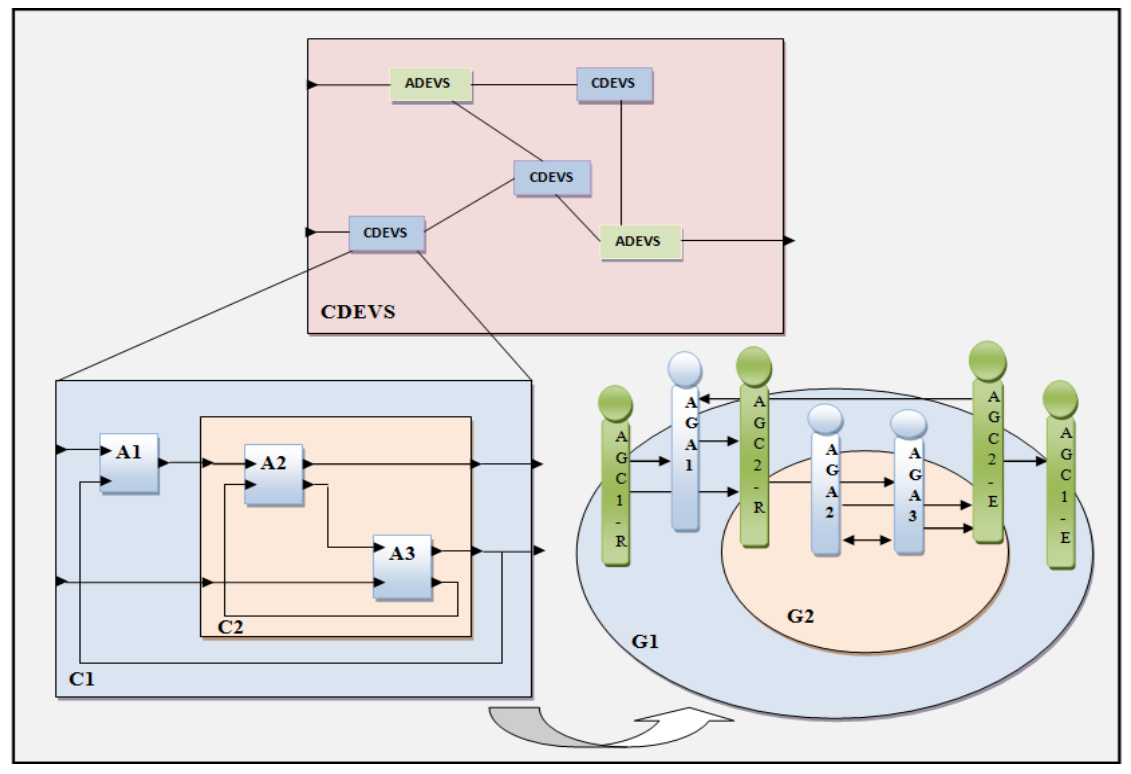

Fig. 3. DEVS/AGR transformation 


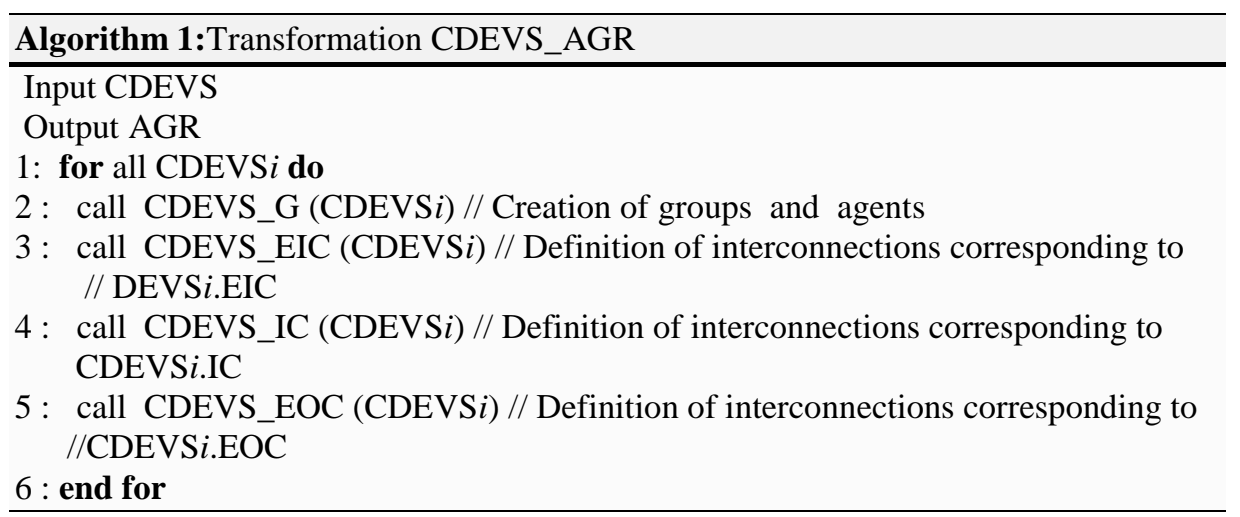

Function 1: ADEVS_AGA ( ADEVS : AtomicDEVS) : AGA

1: Create AGA $j$ // Agent model corresponding to ADEVS $j$

2: Create a mailbox Mb of size $=$ ADEVSj.Inports.Size

3: Create a sending box $\mathrm{Sb}$ of size $=\mathrm{ADEVSj}$.Outports. Size

4: if an external event is appeared in a port:ADEVSj.Inports then

5: Run AGAj. $\mathrm{P}_{\mathrm{a}} / /$ corresponding to ADEVSj. $\delta_{\text {ext }}$

6 : end if

7 : if the life time $t_{a}$ of state $S$ is elapsed then

8: Run AGAj. $\mathrm{F}_{\mathrm{a}}$ // corresponding to ADEVSj. $\delta_{i n t}$

9: Run AGA $j$. Infl $_{\mathrm{a}}$ // corresponding to ADEVS $j . \lambda$

10 : end if
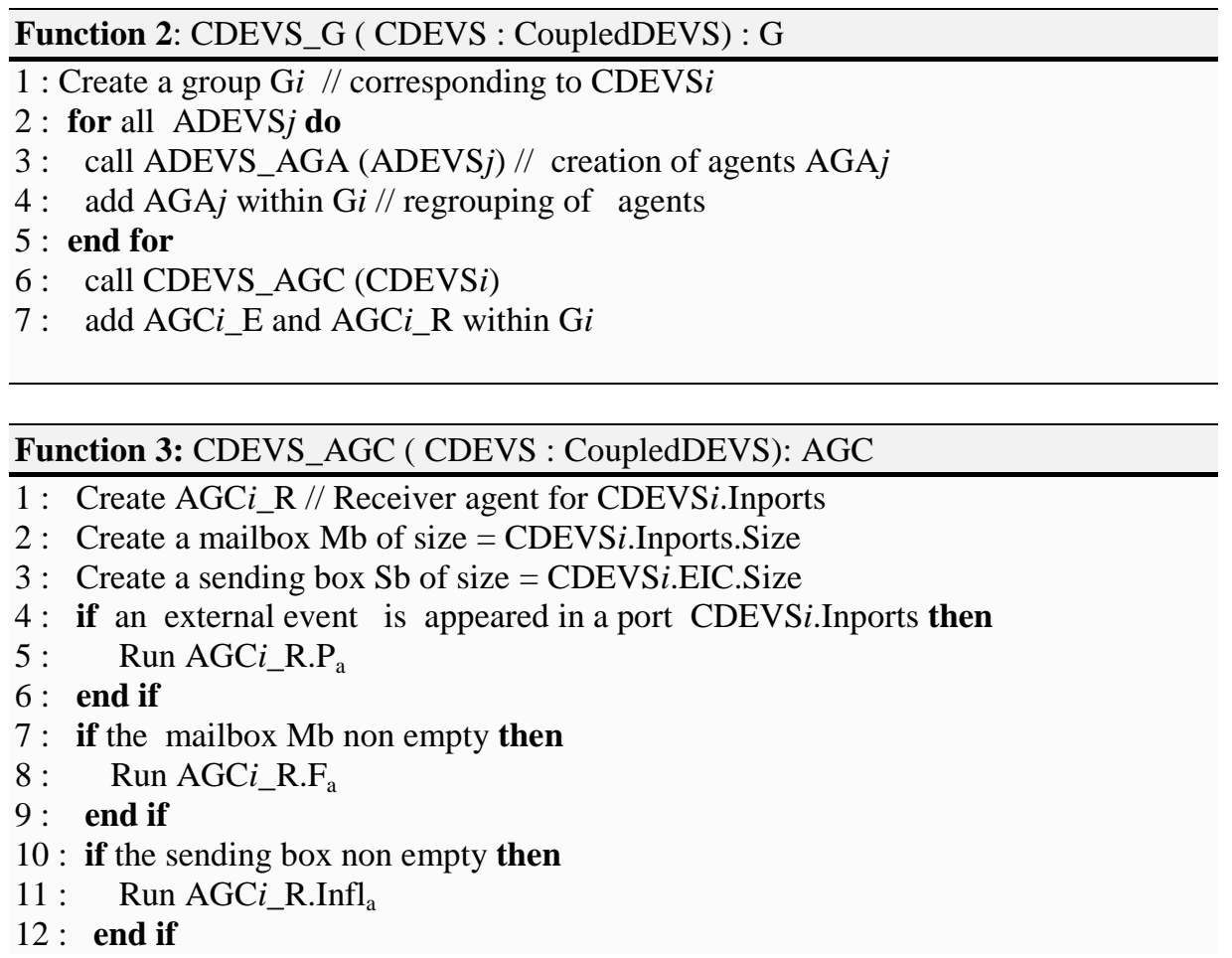
13: Create AGCi_E // emitter agent for CDEVSi.Outports

14: Create a mailbox Mb of size $=$ CDEVS $i$.EOC.Size

15: Create a sending box $\mathrm{Sb}$ of size $=$ CDEVSi.Outports. Size

16: if an external event is appeared in a port: CDEVSi.Outports then

17 : Run AGCi_E.P

18: end if

19: if the mailbox Mb non empty then

20: Run AGCi_E.Fa

21: end if

22: if the sending box non empty then

23: Run AGCi_E.Infl

24: end if

\section{Procedure 1: CDEVS_EIC(CDEVS : CoupledDEVS)}

$1:$ for $m=1$ until the number of CDEVSi.Inports do

2: for $j=1$ until the number of internal models $M$ do

3 : for $k=1$ until the number of $\mathrm{M} j$.Inports do

4: if (CDEVS $i . \mathrm{M} j=\mathrm{ADEVS} j)$ then

5: $\quad$ if $(\mathrm{CDEVS} i \mathrm{X} m=\mathrm{ADEVS} j . \mathrm{X} k)$ then $/ / E I C$ between coupled model and atomic model

6: Define the set of messages between Gi.AGCi_R and Gi.AGAj

7: $\quad$ end if

8: $\quad$ end if

9: if $(\mathrm{CDEVS} i \cdot \mathrm{M} j=\mathrm{CDEVS} j)$ then

10: if (CDEVSi.X $m=$ CDEVSj.Xk) then // EIC between two coupled models

11: Define the set of messages between Gi.AGCi_R and Gj.AGCj_R

12: end if

13: end if

14: end for

15: end for

16 : end for

Procedure 2: CDEVS_IC(CDEVS : CoupledDEVS)

$1:$ for $j=1$ until the number of internal models

M do

2: for $k=1$ until the number of internal Models M do

3: if $j \neq k$ then // an outports should not be coupled with an inports of the same model.

4: for $n=1$ until the number of $\mathrm{Mj}$. Outports do

5: $\quad$ for $m=1$ until the number of $\mathrm{M} k$.Inports do

6: if (CDEVS $i . \mathrm{M} j=\mathrm{CDEVS} j)$ and (CDEVS $i . \mathrm{M} k=\mathrm{CDEVS} k)$ then

7: $\quad$ if $(\mathrm{CDEVS} j$.Y $n=\mathrm{CDEVS} k . \mathrm{X} m)$ then $/ / I C$ between two coupled models

8: $\quad$ Define the set of messages between Gj.AGCj_E and Gk.AGCk_R

9: $\quad$ end if

10: $\quad$ end if

11: if (CDEVS $i \cdot \mathrm{M} j=\mathrm{ADEVS} j)$ and $(\mathrm{CDEVS} i \cdot \mathrm{M} k=\mathrm{ADEVS} k)$ then

12: if (ADEVSj.Y $n=$ ADEVS $k . X m)$ then // IC between two atomic models

13: Define the set of messages between $\mathrm{G} i$.AGA $j$ and Gi.AGA $k$

14: $\quad$ end if 


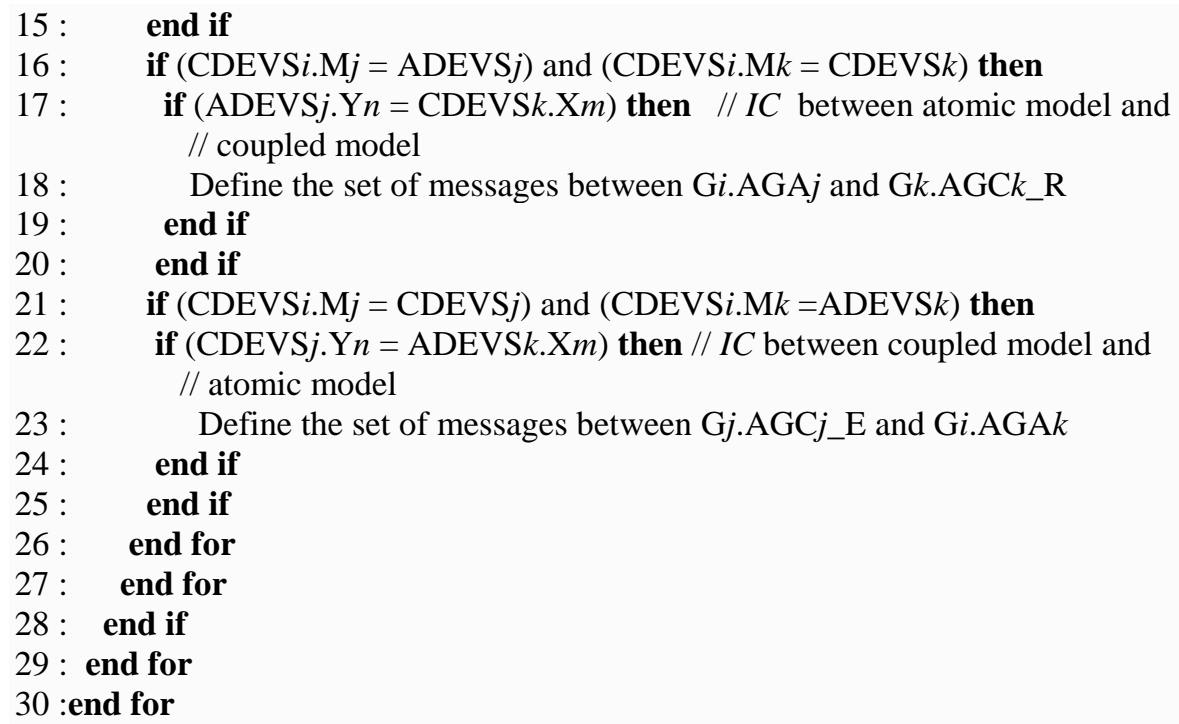

The table 1 below shows a set of passage rules between AGR and DEVS model according to equations (1) and (2) regarding AGR model and equations (3) related to DEVS as well as the algorithms shown above. 
Table 1. Correspondence between DEVS and AGR.

\begin{tabular}{|c|c|c|c|}
\hline \multicolumn{2}{|c|}{ DEVS } & \multicolumn{2}{|r|}{ AGR } \\
\hline \multirow[t]{6}{*}{ Atomic } & $\mathrm{X}$ & \multirow[t]{6}{*}{ Agent $_{a}$} & Percept $_{\mathrm{a}}$ \\
\hline & $\mathrm{Y}$ & & AGC_E \\
\hline & Sext & & $\mathrm{P}_{\mathrm{a}}$ \\
\hline & Sint & & $\mathrm{F}_{\mathrm{a}}$ \\
\hline & $\lambda$ & & $\operatorname{Infl}_{\mathrm{a}}$ \\
\hline & $\mathrm{S}$ & & Agent behaviour (Role) \\
\hline \multirow[t]{6}{*}{ Coupled } & $X$ & \multirow[t]{6}{*}{ Group } & AGC_R \\
\hline & $\mathrm{Y}$ & & AGC_E \\
\hline & M & & $\begin{array}{l}\text { Group / } \mathrm{M} \in \mathrm{CDEVS} \| \\
\text { Agent / } \mathrm{M} \in \mathrm{ADEVS}\end{array}$ \\
\hline & EIC & & $\begin{array}{l}\text { Interconnection between AGCi_R \& AGCj_R / } \\
\operatorname{Mj} \in \operatorname{CDEVS},(i, j) \in N \| A G C i \_R \& \text { AGAj / } \\
\operatorname{Mj} \in \operatorname{ADEVS},(i, j) \in N\end{array}$ \\
\hline & EOC & & $\begin{array}{l}\text { Interconnection between AGCj_E \& AGCi_E / } \\
\text { Mje CDEVS, }(i, j) \in N \| \text { AGAj \& AGCi_E / Mje } \\
\text { ADEVS, }(i, j) \in N\end{array}$ \\
\hline & IC & & 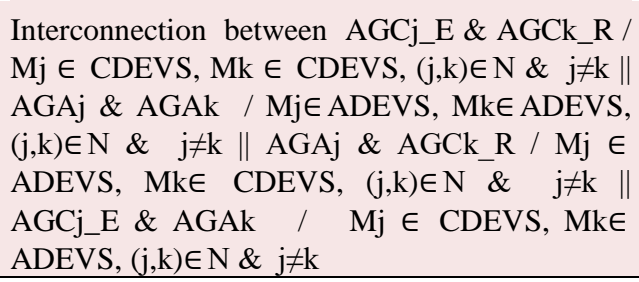 \\
\hline
\end{tabular}

\subsection{Architecture for Simulation System}

The figure 4 presents a global system architecture based on the proposed approach. The interaction module (1) allows the system agent to determinate all coupled and atomic models of system to be simulated through a set of pre-defined models in base of models (2). This base contains a set of re-used models (The system agent can grow it progressively).

The capture of models is done through a set of files code of system simulation. Thus, the system agent may follow the simulation evolutions on the display system (7) by having the possibility to interact with the system thanks to interaction module (1). The parameters are the components of equation of atomic and coupled models. An analysis of data integration is carried out in this level.

The files are saved in XML models (3). Then, the system transforms them thanks to CDEVS/AGR extension (4). The management module of agents (5) allows obtaining a set of agents and groups of agents according to AGR model. Those agents are integrated into MAD-KIT multi-agent platform (8) to obtain the executable code of system simulation. 


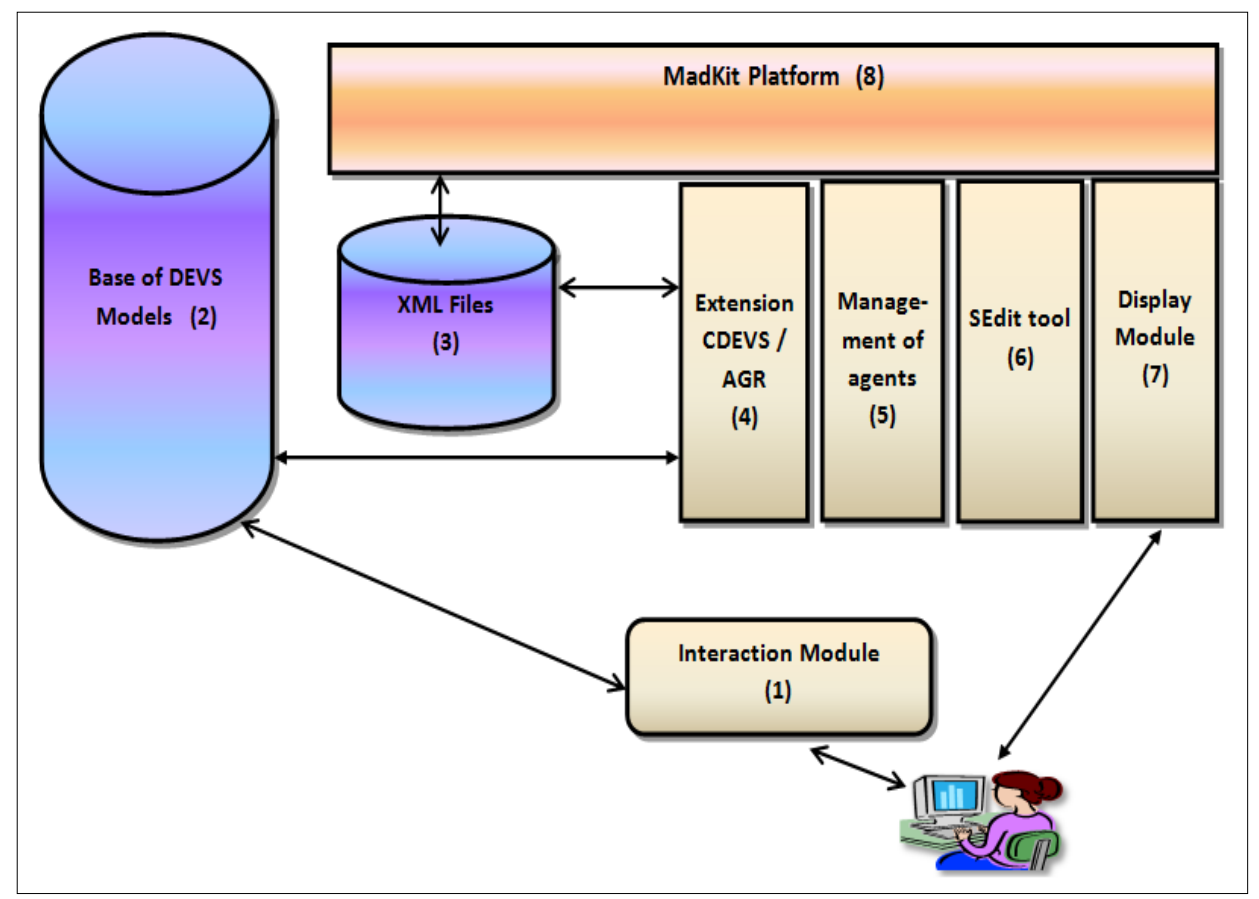

Fig. 4. Overall architecture of the simulation system.

\section{Conclusion}

In this paper we have proposed a new framework for modeling and simulation of complex systems using multi-agent platform MAD-KIT. The proposed approach is based on the DEVS formalism for system modeling and the AGR of MAD-KIT for their simulation. The main idea behind our approach is to take advantage of the formal power of DEVS formalism for verification and validation and the power of the MADKIT platform advanced simulation tools.

The advantage of our approach is that it offers an environment for the design and development of a very flexible and extensible modeling and simulation framework. The later will allow adding DEVS formalism to the modeling formalisms already existing in the platform such as Petri Nets, UML, interaction diagrams, etc. As a perspective, we will focus on testing our extension by modeling and simulating real complex systems such as industrial petroleum systems.

\section{References}

1. Simon, H.A. (1969) The sciences of the artificial, Cambridge (MA). MIT Press.

2. Bertalanffy, L.V. (1968) Théorie générale des systèmes. Dunod.

3. Fishwick, P.A. (1995) Simulation Model Design and Execution. Building Digital Worlds, Prentice Hall.

4. Sonnessa, M. (2004) Modelling and simulation of complex systems (doctoral dissertation, PhD Thesis in "Cultura e impresa", University of Torino, Italy).

5. Shannon, R.E. (1976) Simulation modeling and methodology. Proceedings of the 76 bicentennial conference on Winter simulation.9-15.

6. Oussalah, C. (1998) Modèles hiérachisés multi-vues pour le support de raisonnement dans les domaines techniques. Technical report. 
7. Ingalls, R.G. (2001) Introduction to simulation. Proceedings of the 33nd conference on Winter simulation. IEEE Computer Society, 7- 16.

8. Shannon, R.E. (1998) Introduction to the art and science of simulation. Proceedings of the 30th conference on Winter simulation. IEEE Computer Society Press, 7-14.

9. Fishwick, P.A. (1997) Computer simulation: growth through extension. Transactions of the Society for Computer Simulation International, 14(1), 13-23.

10. Vangheluwe, H. (2008) Foundations of modelling and simulation of complex systems. Electronic communication of the EASST, 10: Graph Transformation and Visual Modeling Techniques. http://eceasst.cs.tuBerlin.de./index.php/eceasst/issue/view/19.

11. Zeigler, B.P., Praehofer, H. and Kim, T.G. (2000) Theory of Modeling and Simulation, Second edition, Academic Press.

12. Gutknecht, O. and Ferber, J. (2000) The MadKit agent plateforme architecture. Laboratoire d'Informatique, Robotique et Microélectronique de Montpellier.

13. Ferber, J. (1995) Les systèmes multi-agents : vers une intelligence collective. Informatique, intelligence Artificielle, Intereditions, Paris.

14. Ferber, J. (1997) Les Systèmes Multi-Agents : Un Aperçu Général, Revue Technique et Science Informatiques, Hermes-Lavoisier.

15. Michel, F., Ferber, J. and Drogoul, A. (2009) Multi-Agent Systems and Simulation : A survey from the agent's community perspective. Multi-Agent systems : simulation and application edited by A. M. Uhrmacher, D. Weyns- CRC Press- Taylor and Francis Group , pp. 3-52.

16. Ferber, J. and Gutknecht, O. (1998) Aalaadin: a meta-model for the analysis and design of organizations in multi-agent systems, ICMAS (International Conference on Multi-Agent Systems), Paris, Y. Demazeau (ed), IEEE Press, pp. 128-135.

17. Seddari, N., Redjimi, M., \& Benoudina, L. (2013, January). Operational approach for modeling and simulation of an industrial process. In 2013 International Conference on Computer Applications Technology (ICCAT) (pp. 1-6). IEEE.DOI: 10.1109/ICCAT.2013.6522031.

18. Seddari, N., \& Redjimi, M. (2013, March). Multi-agent modeling of a complex system. In 2013 3rd International Conference on Information Technology and e-Services (ICITeS) (pp. 1-6). IEEE.

19. Seddari, N., Belaoued, M., \& Bougueroua, S. (2017). Agent/Group/Role Organizational Model to Simulate an Industrial Control System. World Academy of Science, Engineering and Technology, International Journal of Social, Behavioral, Educational, Economic, Business and Industrial Engineering, 11(10), 2376-2385.

20. Seddari, N. and Redjimi, M. (2013). Multi-Agent Modeling of a Complex System . In DLINE Journal - Transactions on Machine Design (TMD), Vol:1 Issue: 2, pp 61-75.

21. Vangheluwe, H. (2001) The Discrete EVent System specification DEVS Formalism. Technical report, 2001.http ://moncs.cs.mcgill.ca/.

22. Barros, F. (1995) Dynamic structure discrete event system specification : a new Formalism for dynamic structure modelling and simulation. In Proceedings of Winter Simulation Conference.

23. Uhrmarcher, A. (2001) Dynamic Structures in Modeling and Simulation : A Reflective Approach. ACM Transactions on Modeling and Computer Simulation vol. 11 2001, pages 206-232.

24. Ntaimo, L. and Zeigler, B.P. (2004) Expressing a forest cell model in parallel DEVS and timed cell-DEVS formalisms. Proceedings of the 2004 Summer Computer Simulation Conference.

25. Troccoli, A. and Wainer, G. (2003) Implementing parallel cell-DEVS. In IEEE, Proceedings of the 36th Annual Simulation Symposium.

26. Davilla, J. and Uzcategui, M. (2000) GALATEA: A multi_agent simulation platform, in international conference on modelling, simulation and neural networks MSNN'2000, Mérida Venezuela.

27. Davilla, J., Gomez, E., Lafaille, K., Tucci, K. and Uzcategui, M. (2005) Multi Agent Distributed Simulation with GALATEA ; Proceedings of the 2005 Ninth IEEE International Symposium on Distributed Simulation and Real-Time Applications (DSRT'05).

28. Mattei, S., Bisgambiglia, P.A., Delhom, M. and Vittori, E. (2012) Towards Discrete Event Multi Agent Platform Specification, Computation Tools 2012 : The third International Conference on Computational Logics, Algebras, Programming, Tools, and Benchmarking, pp. 14-21.

29. Uhrmacher, A.M. and Shattenberg, B. (1998) Agents in Discrete Event Simulation, in Proceedings of ESS98. 
30. Himmelspach, J., Röhl, M. and Uhrmacher, A. M. (2010) Component based modelling and simulation for valid multi-agent system simulations. International journal for Applied Artificial Intelligence, 24(5):414-442.

31. Himmelspach, J. (2012) JAMES II: Extending, Using, and Expeiments, in: Proceedings of the 5 th Simutools Conference ICST, pp 208- 210.

32. Weyns, D., Van Dyke Panurak, H., Michel, F., Holvoet, T. and Ferber, J. (2005) Environments for Multi agent Systems Statec of the art and Research Challenges. In Environments for Multi-agent Systems, edited by D .weyns, H. Dyke Panurak, and F. Michel, Vol 3374 of lecture Notes in Computer Science, 1-47. Berlin, Heidelberg : Springer Berlin Heidelberg.

33. Weyns, D., Schumacher, M., Ricci, A., Viroli, M. and Holvoet, T. (2005) Environments in Multi-agent Systems. The Knowledge Engineering Review, 20 (02).

34. Wooldridge, M. (2009) An introduction to Multi-agent Systems. 2nded. Chistester, UK Jhon Wiley \& Sons.

35. Steiniger, A., Krüger, F. and Uhrmacher, A.M. (2012) Modeling Agents and Their Environment in Multi-level-DEVS, in Proceedings of the Winter Simulation Conference.

36. Ramat, E. and Preux, P. (2003) Virtual Laboratory Environment (VLE): a software environment oriented agent and object for modeling and simulation of complex systems, Simulation and modeling practice and theory, vol 11, $\mathrm{N}^{\circ} 01 \mathrm{pp} .45-55$.

37. Quesnel, G., Duboz, R. and Ramat, E. (2009) The Virtual Laboratory Environment - An operational framework for multi-modelling and analysis of complex dynamical systems, Simulation and modeling practice and theory, vol 17, $\mathrm{N}^{\circ} 4$ pp. 641- 653.

38. Ilachinski, A. (2001) Cellular Automata, a Discrete Universe, World Scientific Publishing Co, ISBN 981-02-4623-4.

39. Wainer, G. A. et Giambiasi, N. (2001) Application of the Cell-DEVS Paradigm for Cell Spaces Modelling and Simulation, Simulation, vol. 76, $\mathrm{N}^{\circ}$ 01, p 22-39.

40. Seddari N., Redjimi, M. and Boukelkoul, S. (2014). Using of DEVS and MAS Tools for Modeling and Simulation of an Industrial Steam Generator, CIT 22, pp.171-189.

41. Ben-Naoum, L., Boel, R., Bongaerts, L., De Schutter B., Peng, Y., Valckenaers, P., Vandewalle, J. and Wertz V (1995). Methodologies for discrete event dynamic systems: A survey, Journal A, 36(4):3-14.

42. Seddari, N. (2015). Outils formels et opérationnels pour la modélisation et la simulation des systèmes complexes (Doctoral dissertation, Université de Skikda). 